\title{
Multiobjective genetic algorithm approach to optimize beam matching and beam transport in high-intensity hadron linacs
}

\author{
M. Yarmohammadi Satri, ${ }^{1,2, *}$ A. M. Lombardi, ${ }^{2}$ and F. Zimmermann ${ }^{2}$ \\ ${ }^{1}$ School of Particles and Accelerators, Institute for Research in Fundamental Sciences (IPM), \\ P.O. Box 19395-5531, Tehran, Iran \\ ${ }^{2}$ CERN, 1211 Geneva 23, Switzerland
}

(Received 28 February 2019; published 22 May 2019)

\begin{abstract}
In this article we demonstrate how a multiobjective genetic algorithm, like the nondominated sorting genetic algorithm II (NSGAII), and a selection tool, like the technique for order preference by similarity to ideal solution (TOPSIS), can be employed for beam matching and for optimizing the beam transport in the low and medium energy section of a modern hadron linac including space charge effect. Combining NSGAII with the particle tracking code TRAVEL v4.07, we determine the Pareto optimal front, and then apply TOPSIS for the final selection using the example of the $160 \mathrm{MeV} \mathrm{H}^{-}$LINAC4 at CERN. We first determine the matching parameters yielding the optimum transport of the $45 \mathrm{keV} \mathrm{H}^{-}$beam from the ion source to the radio-frequency quadrupole (RFQ) and then onwards at the entrance of the drift tube linac (DTL). Next, we optimize six parameters of the beam phase space to maximize the beam transmission from the exit of the RFQ to the DTL through the medium energy transport section. Finally, we benchmark our predictions with simulations based on the independent TRACE 3-D code and also against a beam experiment, in which the transverse emittance was obtained from a temporary slit-and-grid emittance diagnostic device behind the medium energy beam transport line.
\end{abstract}

DOI: 10.1103/PhysRevAccelBeams.22.054201

\section{INTRODUCTION}

The CERN LINAC4 is part of the LHC injector upgrade [1]. It accelerates $\mathrm{H}^{-}$ions with a peak current of $62 \mathrm{~mA}$ up to $160 \mathrm{MeV}$ in an $80 \mathrm{~m}$ long accelerator housed in a tunnel $12 \mathrm{~m}$ underground. The LINAC4 successively consists of a $45 \mathrm{keV}$ rf volume ion source, a two-solenoid low energy beam transport (LEBT), a $352.2 \mathrm{MHz}$ radio-frequency quadrupole (RFQ) accelerating the beam to $3 \mathrm{MeV}$, a medium energy beam transport (MEBT) line, a $50 \mathrm{MeV}$ FFDD periodic lattice drift tube linac (three DTL tanks), a $100 \mathrm{MeV}$ FODO periodic structure cell-coupled drift tube linac (CCDTL), and a FODO periodic lattice pi-mode structure (PIMS) bringing the beam to the final energy of $160 \mathrm{MeV}$ [2]. The MEBT is shown in Fig. 1. The machine is equipped with various temporary and permanent diagnostic tools to monitor the performance over its entire length. The temporary diagnostics bench was placed downstream of the RFQ, MEBT, and the first DTL tank, respectively, during different phases of the LINAC4 low and medium energy

\footnotetext{
*Corresponding author. myarmohammadi@ipm.ir, mahboobeh_yy@yahoo.com

Published by the American Physical Society under the terms of the Creative Commons Attribution 4.0 International license. Further distribution of this work must maintain attribution to the author(s) and the published article's title, journal citation, and DOI.
}

beam commissioning. This diagnostics bench includes a slitand-grid emittance meter device designed for a direct sampling of the horizontal and vertical phase space along with several other diagnostic devices [3]. The slit-and-grid emittance measurement has an error of order $\pm 10 \%$.

During the period of the LINAC4 low and medium energy beam commissioning, the beam peak current was lower than the ultimate design value $(62 \mathrm{~mA})$. That is, the beamenvelope matching, beam matching, and emittance measurements were performed with $15 \mathrm{~mA}$ peak current for the LEBT and $10 \mathrm{~mA}$ for the MEBT. The beam transmission through the RFQ was about $70 \%$, and hence noticeably smaller than the design value of $95 \%$. According to measurements and simulations, the low transmission was due to the fact that, during the beam commissioning, the emittance at the entrance of the RFQ greatly exceeded the design value. Namely, the normalized total emittance of $3.6 \pi \mathrm{mm} \mathrm{mrad}$, measured in the LEBT, was about 1.3 times larger than the RFQ total acceptance, $2.6 \pi \mathrm{mm} \mathrm{mrad}$ in both transverse planes [4] and [5]. Beyond that the normalized total emittance of the MEBT beam at entrance of the DTL, $6.1 \pi \mathrm{mmmrad}$ and $3.8 \pi \mathrm{mm}$ mrad, is about 2.5 and 4 times smaller than the DTL total acceptance, about $15 \pi \mathrm{mm} \mathrm{mrad}$, in horizontal and vertical transverse planes, respectively.

In the initial phase of the beam commissioning, one needs to match the beam to the optics of the accelerating structures in the presence of space charge. This requires the 


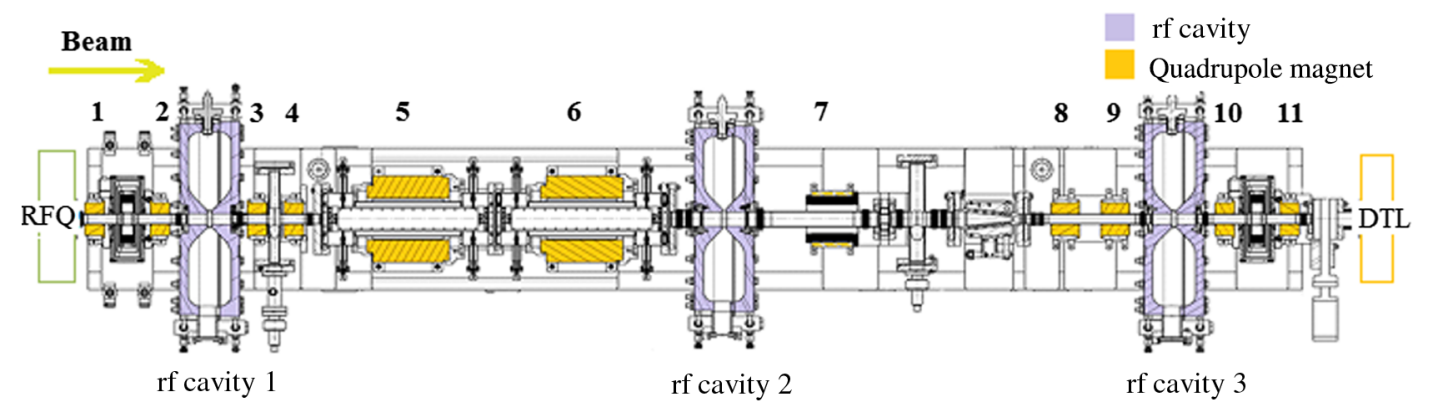

FIG. 1. Layout of the $3 \mathrm{MeV}$ MEBT line [3], with knob numbers shown at the top.

matching of the beam Twiss parameters according to the actual beam current, e.g. $10 \mathrm{~mA}$ for the LINAC4 DTL semiperiodic structure during the medium-energy beam commissioning. The matched envelope for the periodic lattice of an accelerating structure refers to the quasiperiodic evolution of the rms beam size along the structure with an approximately constant average value for the two transverse directions [1] and [6]. This confined evolution of the beam envelope is a desired precondition for the matching and commissioning of a sequence of long and multiple accelerating structures such as those in LINAC4.

Usually, the beam dynamics and the commissioning optimization of a beam passing through an accelerating structure necessitates a start-to-end simulation with consideration of the transmission, the beam-envelope matching for each of the subaccelerating structures, and the transport and beam matching $(\mathrm{BM})$ in between the substructures. However, the space charge forces are nonlinear in nature and the accelerators include several adjustable components, for instance solenoids, quadrupole magnets, and rf cavities. In this situation, the optimal performance of the complex can be obtained by fulfilling a set of objectives while respecting practical constraints like hardware limitations (e.g. the maximum field strength).

The NSGAII multiobjective genetic algorithm, allowing for several variables, objectives, and constraints, has previously been used for addressing problems in accelerator physics [7-11], in optimal control [12], and in engineering [13-15]. This paper extends its application to the beam matching and commissioning process for the CERN LINAC, and any similar hadron accelerator.

Through this article we attempt to illustrate how the NSGAII multiobjective genetic algorithm and TOPSIS provided us with answers to questions such as the following: How can the NSGAII and TOPSIS be used for beam matching and specifically beam-envelope matching (BEM)? How can the beam-envelope matching be applied for a periodic accelerating structure? How much can the matching performance be enhanced by applying the NSGAII algorithm and TOPSIS?

Our article is structured as follows.

In Sec. II we review the beam envelope equation, the relation defining the beam matching, and the logics behind the development of NSGAII multiobjective genetic algorithm strategy along with the criteria for reaching the objectives. TRACE 3-D and other codes traditionally used for beam matching find a local optimum and the best solution depending on the initial seed. By contrast NSGAII determines the globally optimum solution.

In Secs. III and IV of this paper we present the procedure and the NSGAII setup for the beam matching to the RFQ and the beam-envelope matching into the DTL with 15 and $10 \mathrm{~mA}$ current, respectively.

In Sec. V, the NSGAII result for the beam matching from the MEBT to DTL with $10 \mathrm{~mA}$ peak current is benchmarked against simulation data from the TRACE 3-D code [16] and compared with the corresponding measurements of the beam transverse phase space.

Section VI draws some conclusions.

\section{BEAM ENVELOPE AND THE NSGAII BEAM MATCHING STATEGY}

The beam-envelope matching is an important task for both the beam dynamics design and for the commissioning of a new hadron linac. It minimizes beam loss and supports the following steps of downstream commissioning.

We briefly recall the theoretical description of the beam evolution and report the physics computation criteria applied for this optimization.

In the absence of collective or nonlinear effects, and absence of acceleration, the transverse evolution of the beam particles is described by Hill's equation. The particle motion in a 2D phase space traces an ellipse such (1) for the $x-x^{\prime}$ phase space (see Fig. 2):

$$
\gamma(z) x^{2}+2 \alpha(z) x x^{\prime}+\beta(z) x^{\prime 2}=\varepsilon
$$

where $\alpha(z), \beta(z)$, and $\gamma(z)$ are called Twiss parameters and the derivation with respect to the longitudinal coordinates is presented as prime.

In periodic accelerating structures, the acceleration changes the concept of a "fully" periodic lattice to a system called semiperiodic. The purpose of the matching is to avoid too large a beam at some location accompanied by a too small beam at some other location. The so-called 


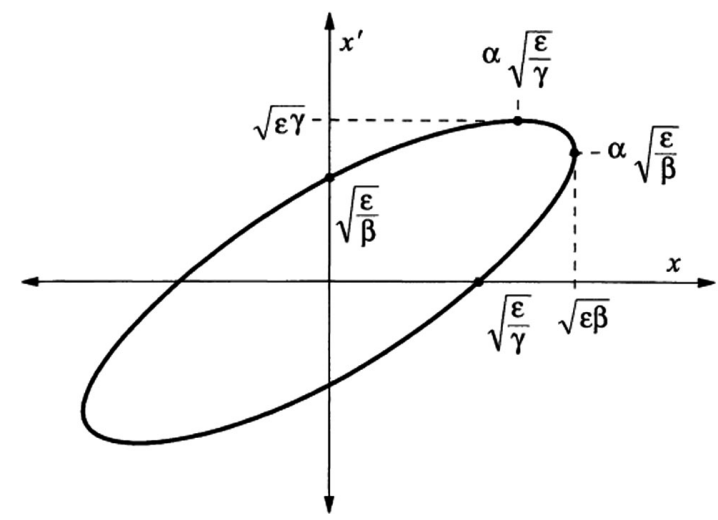

FIG. 2. The phase ellipse of particles in the transverse $x-x^{\prime}$ phase space [17].

beam matching here means that the beam ellipse agrees with the design Twiss parameters. The mismatching factor, MMF, represents the amount of difference between the targeted envelope $\left(\alpha_{m} \alpha_{m}, \beta_{m}\right.$, and $\left.\gamma_{m}\right)$ and the propagated measured envelope $(\alpha, \beta$, and $\gamma)$ :

$$
\begin{gathered}
\gamma_{m} x^{2}+2 \alpha_{m} x x^{\prime}+\beta_{m} x^{\prime 2}=\varepsilon, \\
\gamma x^{2}+2 \alpha x x^{\prime}+\beta x^{\prime 2}=\varepsilon, \\
\Delta=\Delta \alpha^{2}-\Delta \beta \Delta \gamma \text { and } \\
\mathrm{MMF}=\left[1+\frac{\Delta+\sqrt{\Delta(\Delta+4)}}{2}\right]^{1 / 2}-1,
\end{gathered}
$$

where (2) and (3) represent, respectively, the matched ellipse and a mismatched beam ellipse, $\Delta \alpha=\alpha-\alpha_{m}$, $\Delta \beta=\beta-\beta_{m}=\Delta \gamma=\gamma-\gamma_{m}$. The parameter MMF is the mismatch factor [6].

We combined the NSGAII, proposed by Deb et al. as the most effective genetic algorithm [18], and the particle tracking in TRAVEL v4.07, to simultaneously optimize the MMF in one or several planes and the beam transmission (BT). The tracking code TRAVEL v4.07 simulates the particle acceleration process in 3D. One of the simulations results is the rms beam size of the tracked particles along the beam line [19]. As input to the tracking, an empirical beam distribution was chosen $[4,3,20]$. The results of the NSGAII process, representing possible optimal compromises between the multidecision-variable constraints and objective criteria, define the so-called Pareto optimal front.

The multiobjective optimization aims at concurrently maximizing or minimizing the set of objectives while respecting some constraints and limited parameter ranges for different variables and objectives. A general optimization problem is the minimization of a set of $n$ functions $\left\{f_{1}(X), f_{2}(X), \ldots, f_{n}(X)\right\}$ where $X$ denotes the group of $m$ variables to be adjusted $X=\left\{x_{1}, x_{2}, \ldots, x_{m}\right\}$, within certain limits $x_{j}^{\min } \leq x_{j} \leq x_{j}^{\max }$ for $1 \leq j \leq m$. For maximizing some objective, its negative or its inverse can be minimized, equivalently. In general, a solution which minimizes all the functions $f_{i}(1 \leq I \leq n)$ simultaneously does not exist. Among the set of all possible solutions defined by the constraints imposed on the variables $x$ and functions $f$, the Pareto optimal front represents a special subset. $X^{1}$ is called a member of the Pareto optimal solution if there exists no other feasible $X^{2}$ with $f_{i}\left(X^{1}\right)<f_{i}\left(X^{2}\right)$ for one $i$ and $f_{i}\left(X^{1}\right)<f_{i}\left(X^{2}\right)$ for all other $i$; further details can be found in Refs. [18] and [12].

Simulations with different, increasing numbers of generations and populations are executed to create a Pareto optimal front and to confirm its convergence. Following this, the best solution is selected by TOPSIS, as a trade-off among the various solutions on the Pareto optimal front. TOPSIS is a multiple-attribute decision-making method. TOPSIS defines a positive and negative ideal solution, based on weights assigned to the different objectives. It then chooses the Pareto-optimal-front solution closest to the positive and farthest away from the negative ideal solution. More details can be found in Refs. [21] and [22].

To reduce the NSGAII computation time and simplify the process, we organized a step-by-step NSGAII optimization and used the result of one section for continuing with the next section: regarding the two LEBT variables (solenoid 1 and solenoid 2), the BM NSGAII optimization was restricted to the minimization of two objectives $\left(\mathrm{MMF}_{x}\right.$ and $\mathrm{MMF}_{y}$ ) along with the maximization of the beam transmission (BT). The DTL matching was executed with four variables and three objectives (related to the quantities $\mathrm{A}_{x}, \mathrm{~A}_{y}$ defined in Sec. IV, and to BT). The matching from MEBT to DTL was achieved by optimizing six variables with the objective of simultaneously minimizing $\mathrm{MMF}_{x}$, $\mathrm{MMF}_{y}, \mathrm{MMF}_{z}$, and maximizing BT.

We initially studied the targeted minimum or maximum with relaxed constraints by only considering the power limitations of the variable elements (the maximum current or voltage for the rf cavities, solenoids, quadrupoles etc.). The final Pareto optimal front was derived with tighter constraints requiring the solution to stay close to the initial variables.

At each step, the Pareto optimal front provides multiple matching solutions. Considering the robustness of the solution as a practically important aspect, we preferred solution sets with better stability against changes of initial Twiss parameters, of the magnet strength, or of the rf power, and with smoother optical functions.

\section{RFQ BEAM MATCHING}

The LEBT consists of two solenoid magnets. The ultimate goal of the LEBT is propagating and matching 


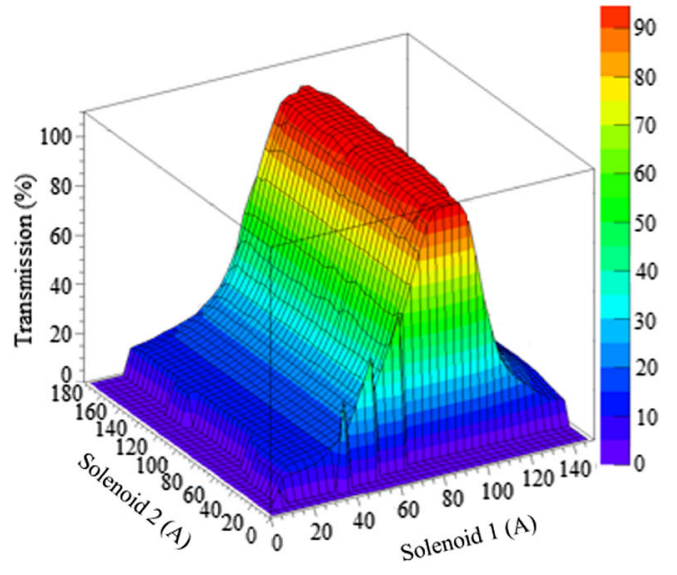

(a)
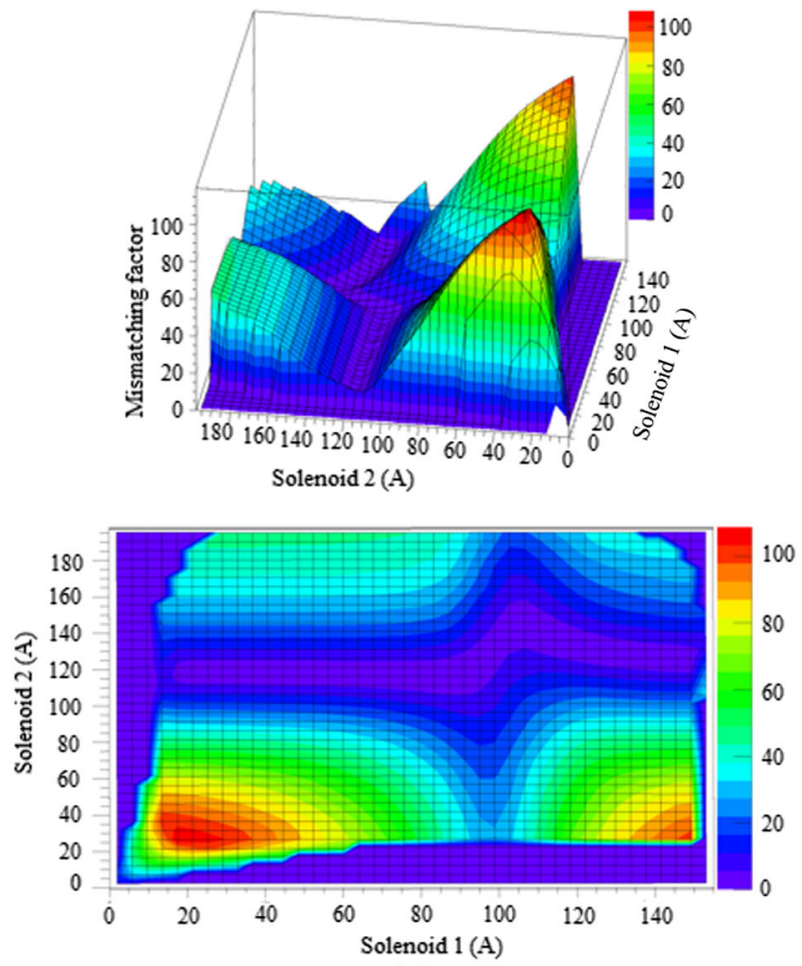

(b)

FIG. 3. (a) Beam transmission and (b) (top) mismatching factor to the RFQ ( $z$ axis) as a function of the solenoid magnet 1 strength ( $x$ axis) and solenoid magnet 2 strength ( $y$ axis); (bottom) mismatching factor is color coded. The space charge effect considered the full current at the time of the beam commissioning $(15 \mathrm{~mA})$.

the beam from the $\mathrm{H}^{-}$source to the RFQ, symmetrically in the horizontal and vertical planes, by choosing the proper settings for the solenoids [23]. To effectively reduce the clock time of NSGAII computation, we restricted the region of solenoid strengths, to an area of values, as shown in Fig. 3. The region of interest was roughly predetermined by linking TRAVEL v4.07 and the Delta code, which is an external error-study module used in conjunction with TRAVEL v4.07 to investigate the effect of beam line errors on the output beam [24]. The simulations use an empirical beam distribution consisting of 209351 macroparticles with the $\mathrm{H}^{-}$rest mass of $0.93926 \mathrm{GeV} / \mathrm{c}^{2}$ and an average kinetic energy of $45 \mathrm{keV}$. The parameters of the beam distribution are summarized in Table I [4].

The goal of our optimization was to minimize the transverse mismatching factors $\left(\mathrm{MMF}_{x}\right.$ and $\left.\mathrm{MMF}_{y}\right)$ and to maximize the transmission from the source exit to RFQ. We ran NSGAII for optimizing the LEBT parameters with 50 generations and 70 populations. The objectives and variable constraints for matching the beam current available at the time of the beam commissioning $(15 \mathrm{~mA})$ are set as follows (the $145 \mathrm{~A}$ current value of the solenoid was scaled to (1): $\mathrm{MMF}_{x}<2, \mathrm{MMF}_{y}<2$, BT $>85 \%, 0.6<$ solenoid 1 scaling factor $<0.73,0.72<$ solenoid 2 scaling factor $<1.06$.

Figure 4 shows the set of the solutions for minimization of $\mathrm{MMF}_{x}$ and $\mathrm{MMF}_{y}$ and maximization of the transmission plotted as the Pareto optimal front of the solution. The Topsis selected set with consideration of the same weights for all the objectives is $\mathrm{MMF}_{x}=0.274, \mathrm{MMF}_{y}=$ 0.257 , and $\mathrm{BT}(\%)=89.144$ and equivalent values of the parameters of the set are solenoid $1=87.25 \mathrm{~A}$ and solenoid $2=115.86 \mathrm{~A}$.

TABLE I. Beam parameters of the used empirical beam distribution for the RFQ beam matching simulation.

\begin{tabular}{cccc}
\hline \hline & $X-X^{\prime}$ & $Y-Y^{\prime}$ & $Z-Z^{\prime}$ \\
\hline Normalized rms emittance & $0.83 \pi \mathrm{mmmrad}$ & $0.79 \pi \mathrm{mm} \mathrm{mrad}$ & $14.89 \pi \mathrm{deg} \mathrm{keV}$ \\
$\beta$ & $1.52 \mathrm{~mm} / \pi \mathrm{mrad}$ & $1.52 \mathrm{~mm} / \pi \mathrm{mrad}$ & $1475.65 \mathrm{deg} / \pi \mathrm{keV}$ \\
$\alpha$ & -5.62 & -5.62 & -0.64 \\
\hline \hline
\end{tabular}



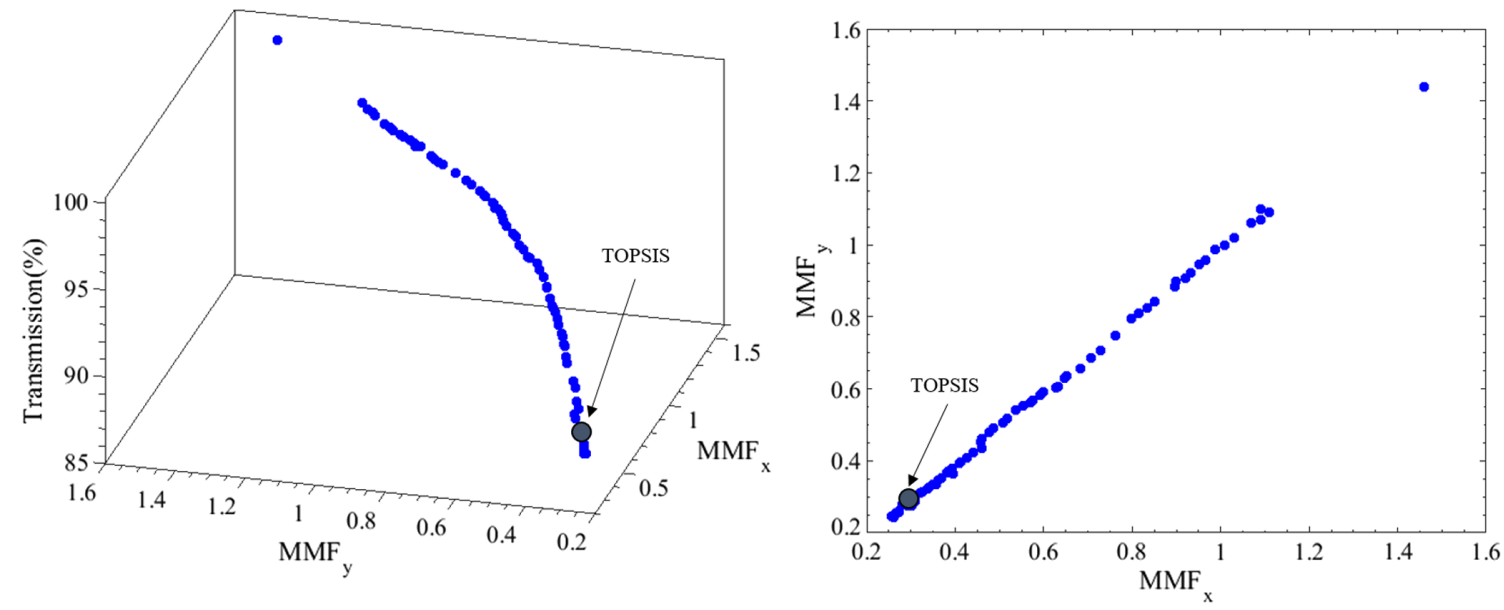

FIG. 4. Pareto optimal front of the LEBT NSGAII optimization and the TOPSIS selected point. Mismatching factor along the transverse $x$ direction versus mismatching factor along the transverse $y$ direction (left), and (right) the transmitted particles to RFQ entrance as a function of $x$ and $y$ transverse plane mismatching factors.

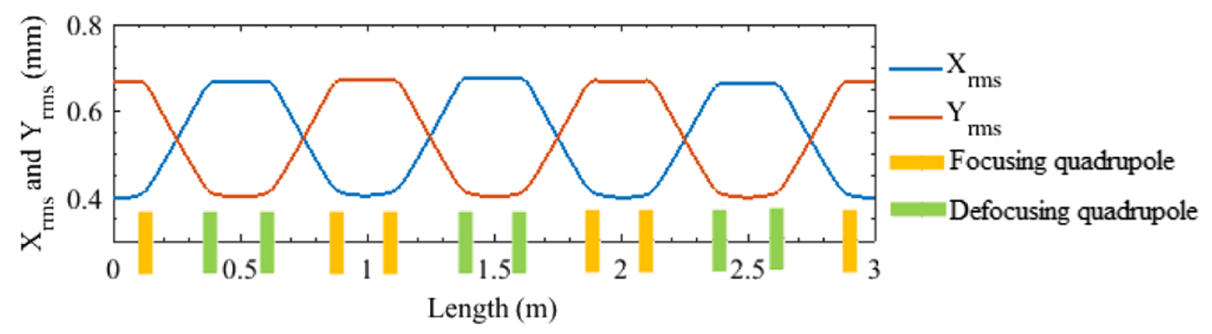

FIG. 5. Typical FFDD structure.

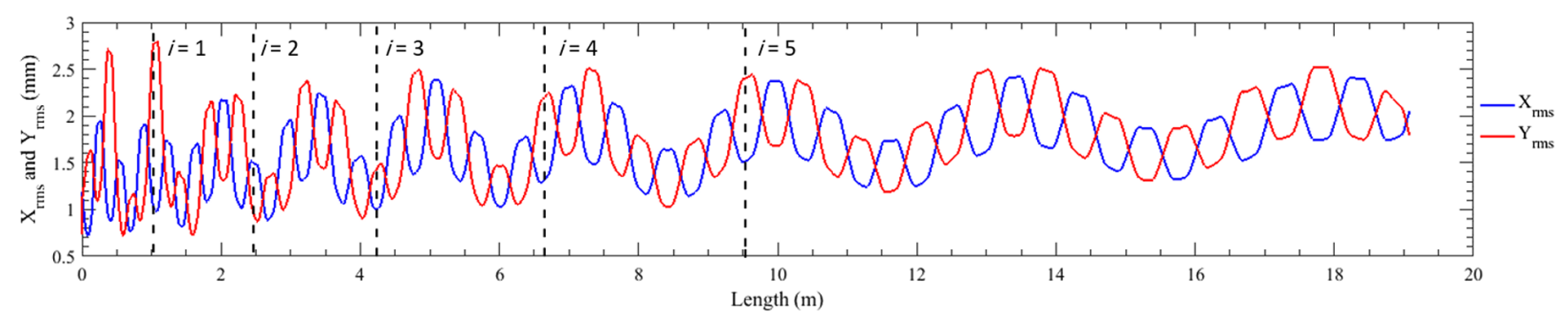

FIG. 6. Beam envelope propagation along the DTL for unmatched Twiss parameters.
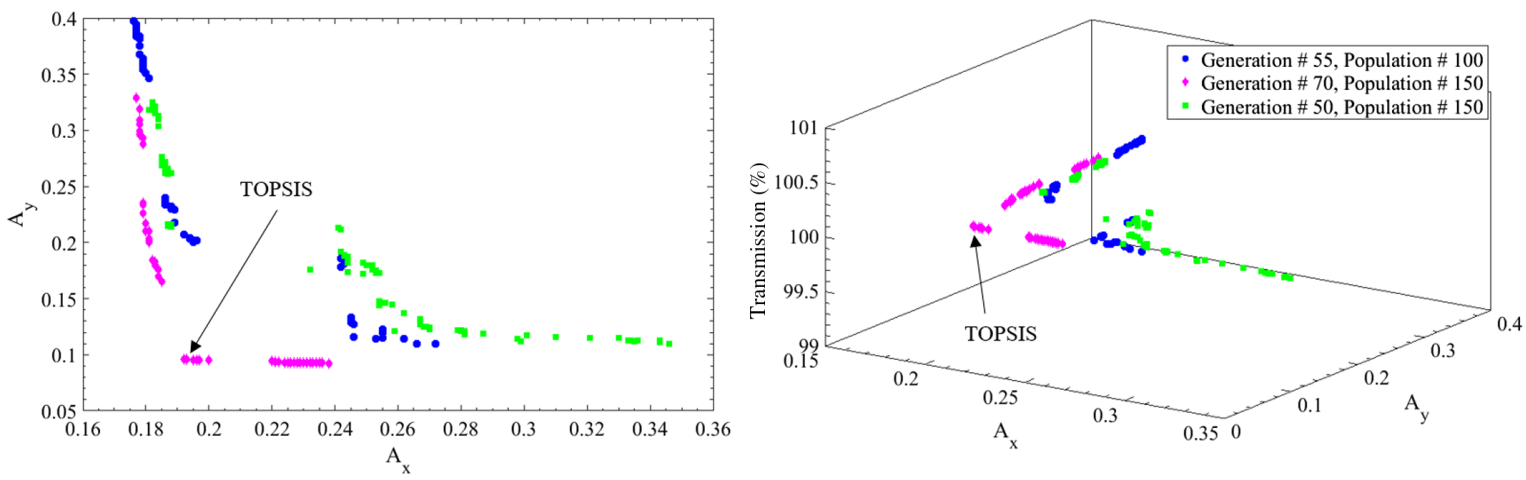

FIG. 7. Pareto optimal front of the last generations and the TOPSIS selected point. Two of the objectives: rms of beam envelope slope between two consecutive quadrupoles of identical polarity along the DTL in the two transverse planes, $A_{x}$ and $A_{y}$ (left). Three of the objectives, $A_{x}, A_{y}$, and the transmission along the DTL (right). 
TABLE II. Beam parameters of the used input beam for the DTL rms envelope matching NSGAII simulation.

\begin{tabular}{cccc}
\hline \hline & $X-X^{\prime}$ & $Y-Y^{\prime}$ & $Z-Z^{\prime}$ \\
\hline Normalized rms emittance & $0.36 \pi \mathrm{mmmrad}$ & $0.35 \pi \mathrm{mmmrad}$ & $175.59 \pi \mathrm{deg} \mathrm{keV}$ \\
$\beta$ & $0.48 \mathrm{~mm} / \pi \mathrm{mrad}$ & $0.39 \mathrm{~mm} / \pi \mathrm{mrad}$ & $0.43 \mathrm{deg} / \pi \mathrm{keV}$ \\
$\alpha$ & 3.64 & -1.25 & 0.013 \\
\hline \hline
\end{tabular}

\section{DTL RMS ENVELOPE MATCHING}

The FFDD is a focusing lattice consisting of four quadrupoles with identical strength and opposite polarity separated by drifts as shown in Fig. 5. And slope of the beam envelope, $\alpha$, between two consecutive quadrupoles of identical polarity is zero. Acceleration in the DTL necessitates the gradual increase of the FFDD quadrupole strength and magnet spacing along the structure, and converting to a semi-FFDD structure. We are interested in smoothly propagating the beam along the structure while avoiding any nondesired betatron oscillation.

The criterion we have applied to suppress the beam envelope betatron oscillation is inspired from the periodic FFDD structure, Fig. 5. Namely, we have set as the objective of the NSGAII algorithm that the square root of the rms beam envelope slope, $\alpha$, between two consecutive quadrupoles of identical polarity approaches zero, as closely as possible, at five different locations along the DTL $\left(A_{x}=\sqrt{\sum_{i=1}^{5} \alpha_{x i}}\right.$ and $\left.A_{y}=\sqrt{\sum_{i=1}^{5} \alpha_{y i}}\right)$ with the goal of simultaneously minimizing $\mathrm{A}_{x}$ and $\mathrm{A}_{y}$ and maximizing the $\mathrm{BT}$ along the structure, Fig. 6 . The longitudinal beam parameters at the DTL input are assumed to be equal to design values for nominal DTL current $(\alpha=-0.0098$ and $\beta=0.2992 \mathrm{deg} / \pi \mathrm{keV}$ ), which is achieved by the matching of the MEBT rf cavities. In the following, we list the conditions for the NSGAII optimization for the DTL beta matching objectives, where $\mathrm{A}_{x}$ and $A_{y}$ are objectives and $\alpha_{x}, \alpha_{y}, \beta_{x}, \beta_{y}$ are variable constraints: $A_{x}<$ $0.4, A_{y}<0.4$, BT $>90 \%, 1<\alpha_{x}<3,-3<\alpha_{y}<-1$, $0.0001<\beta_{x}<0.5,0.0001<\beta_{y}<0.5$.

We used several number of generation and population and the sufficient numbers were achieved with 70 and 150 as generation and population, respectively. The Pareto optimal front represents the convergence of the solutions against a set of possible compromises between $A_{x}, A_{y}$ and the BT. Figure 7 shows the distribution of the solution of two objectives $\left(A_{x}\right.$ and $\left.A_{y}\right)$ while the BT objective is satisfied with $100 \%$ BT for all of the solutions. The selected solution by TOPSIS has the following parameters: $A_{x}=$ $0.192, A_{y}=0.096, \mathrm{BT}(\%)=100, \alpha_{x}=2.439, \alpha_{y}=-1.186$, $\beta_{x}=0.317 \mathrm{~mm} / \mathrm{mrad}, \beta_{y}=0.262 \mathrm{~mm} / \mathrm{mrad}$.

The particles are simulated at $3 \mathrm{D}$ to handle the beam acceleration and the empirical beam distribution consisting of 34503 macroparticles with the $\mathrm{H}^{-}$rest mass of $0.93926 \mathrm{GeV} / \mathrm{c}^{2}$ and an average kinetic energy of $3 \mathrm{MeV}[3,20]$. The parameters of the beam distribution are summarized in Table II.

The propagation of the TOPSIS selected solutions into the DTL is plotted in Fig. 8. The behavior of the rms beam envelope in the DTL resembles the desired periodic FFDD lattice. This solution was used for defining the objectives of the downstream MEBT matching, reported in the following section.

\section{OPTIMAL MEBT CONDITION}

Since the beam ellipse at the exit plane of the RFQ does not have the proper rms size and ellipse orientation to match the desired DTL optics, a matching section is required. The MEBT, Fig. 1, consisting of 11 quadrupoles and $3 \mathrm{rf}$ cavities, is devoted to accomplishing this matching manipulation in the longitudinal and transverse planes, for preparing the beam injection into the DTL. The targetedDTL BEM has been identified from the Pareto optimal front in Sec. IV.

The necessary tools for matching the RFQ beam to the DTL are four quadrupoles and two rf cavities. In other words, the number of knobs available in the MEBT is larger than the minimum required. Using the NSGAII algorithm to find a solution that simultaneously minimizes the three mismatch factors $\mathrm{MMF}_{x}, \mathrm{MMF}_{y}, \mathrm{MMF}_{z}$ and maximizes the beam transmission, as four objectives, the last four quadrupole magnets and the last two rf cavities, Fig. 1, were chosen

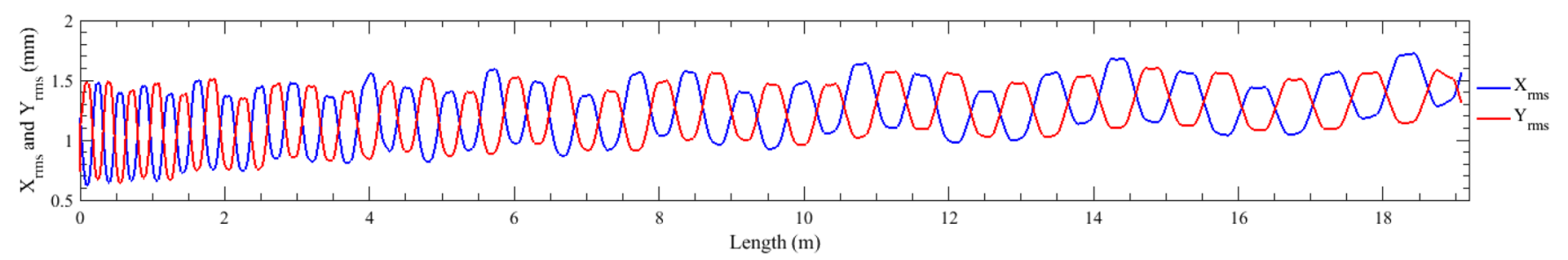

FIG. 8. Propagation of the beam envelope into the DTL for the chosen solution from the Pareto optimal NSGAII for the DTL matching. 

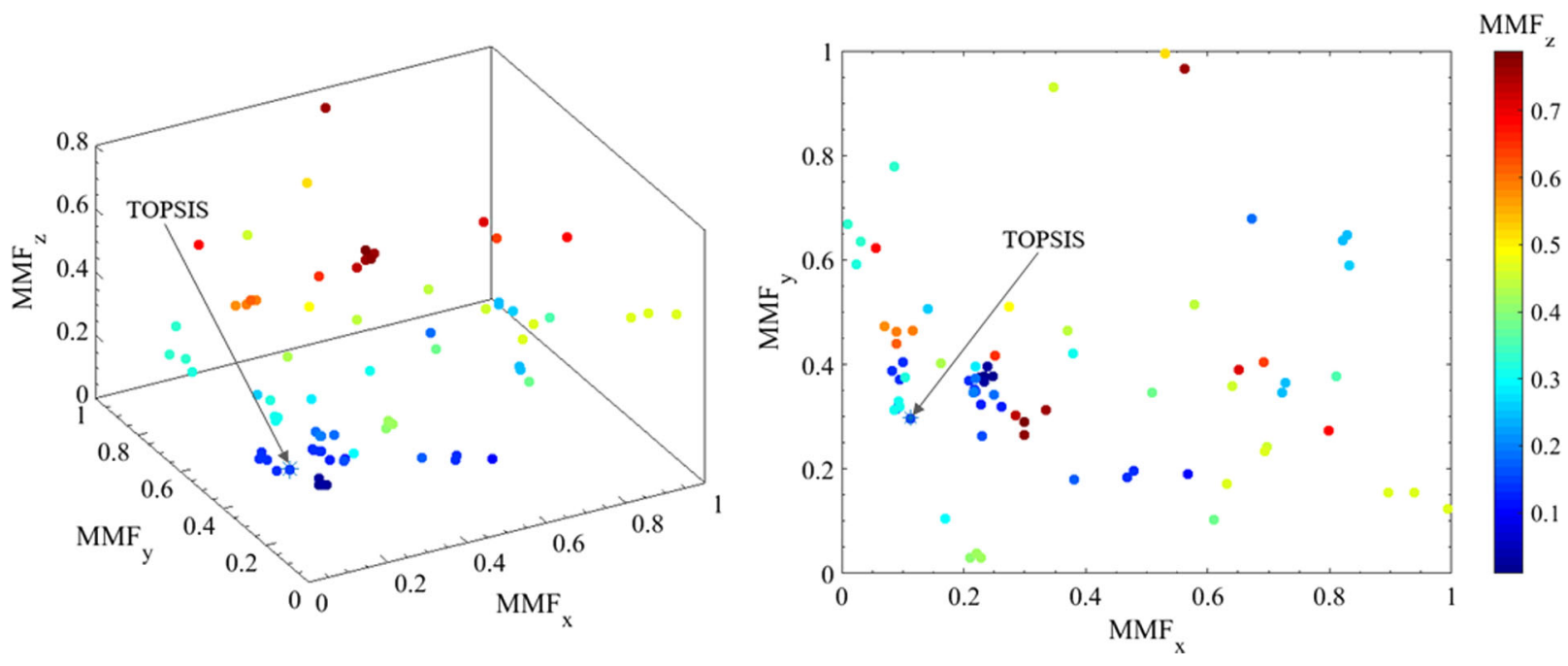

FIG. 9. Pareto optimal front of the last generations and the TOPSIS selected point. Mismatching factor along the transverse $x$ direction versus mismatching factor along the transverse $y$ direction (right), and (left) the longitudinal $z$ direction as a function of $x$ and $y$ transverse plane mismatching factors while the transmission is more than $94 \%$.
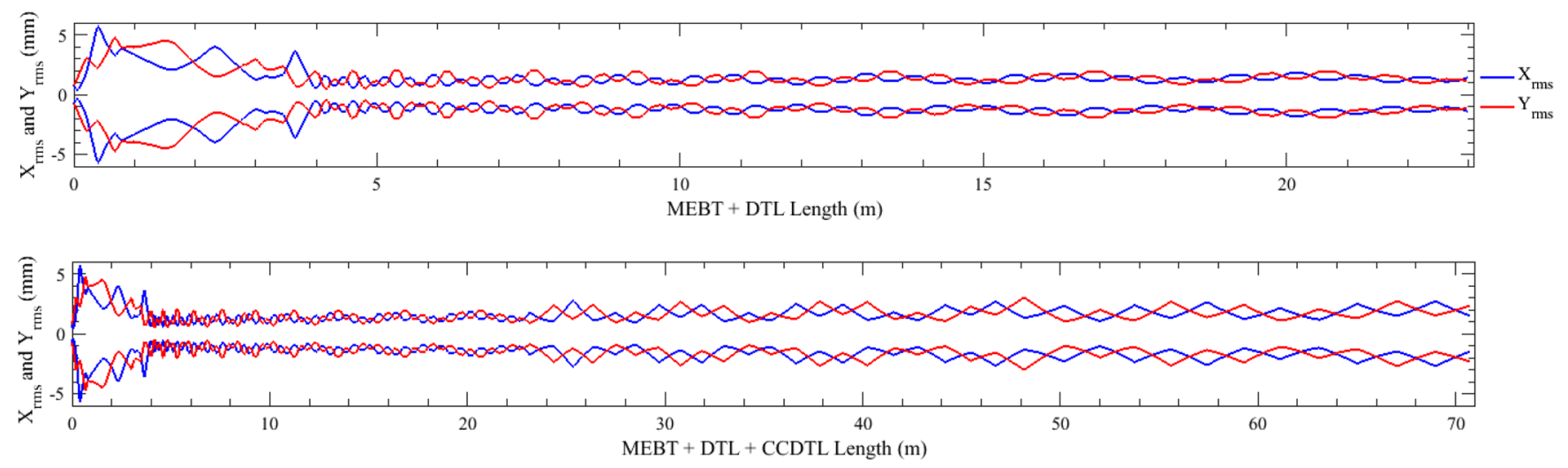

FIG. 10. Beam tracking from RFQ output plane to the end of LINAC4 structure, MEBT + DTL (top), MEBT + DTL + CCDTL + PIMS (bottom).

as variables to be optimized, with the following constraints: $\mathrm{MMF}_{x}<1, \mathrm{MMF}_{y}<1, \mathrm{MMF}_{z}<1, \mathrm{BT}>94 \%, 0.03<$ rf cavity $2<0.09 \mathrm{MV}, \quad 6<$ quadrupole $8<11(\mathrm{~T} / \mathrm{m})$, $-11<$ quadrupole $9<-7(\mathrm{~T} / \mathrm{m}), \quad 0.07<$ rf cavity $3<$ $0.12 \mathrm{MV}, \quad 20<$ quadrupole $10<27(\mathrm{~T} / \mathrm{m}), \quad-31<$ quadrupole $11<-25(\mathrm{~T} / \mathrm{m})$.

The distribution of solutions resulting from a compromise among $\mathrm{MMF}_{x}, \mathrm{MMF}_{y}$, and $\mathrm{MMF}_{z}$ and the TOPSIS selection is plotted in Fig. 9. The specifications of the selected solution are as follows: $\mathrm{MMF}_{x}=0.113, \mathrm{MMF}_{y}=0.298$, $\mathrm{MMF}_{z}=0.149$ and $\mathrm{BT}=95.344(\%)$, rf cavity $2=$ $-0.066 \mathrm{MV}$, quadrupole $8=9.525 \mathrm{~T} / \mathrm{m}$, quadrupole $9=-7.698 \mathrm{~T} / \mathrm{m}$, rf cavity $3=-0.081 \mathrm{MV}$, quadrupole $10=22.230 \mathrm{~T} / \mathrm{m}$, quadrupole $11=-27.796 \mathrm{~T} / \mathrm{m}$.

Then the beam tracks from the RFQ exit plane to the end of LINAC4 (MEBT, DTL, CCDTL and PIMS, respectively). Figure 10 shows the selected condition reasonably well fulfills the matching condition for the upstream semiperiodic lattice. In addition, according to the
TABLE III. Comparison of solutions from TRACE 3-D and from the joined NSGAII and TOPSIS method. Shown are the values of six transverse and longitudinal MEBT knobs, optimized by either program, for matching the $10 \mathrm{~mA} R F Q$ output beam to the DTL. The knob numbers are indicated in Fig. 1.

\begin{tabular}{lcc}
\hline \hline & NSGAII-TOPSIS & TRACE 3-D \\
\hline rf cavity 2 & $-0.066 \mathrm{MV}$ & $-0.065 \mathrm{MV}$ \\
Quadrupole 8 & $9.525 \mathrm{~T} / \mathrm{m}$ & $8.690 \mathrm{~T} / \mathrm{m}$ \\
Quadrupole 9 & $-7.698 \mathrm{~T} / \mathrm{m}$ & $-8.351 \mathrm{~T} / \mathrm{m}$ \\
rf cavity 3 & $-0.081 \mathrm{MV}$ & $-0.089 \mathrm{MV}$ \\
Quadrupole 10 & $22.230 \mathrm{~T} / \mathrm{m}$ & $23.857 \mathrm{~T} / \mathrm{m}$ \\
Quadrupole 11 & $-27.796 \mathrm{~T} / \mathrm{m}$ & $-27.962 \mathrm{~T} / \mathrm{m}$ \\
\hline \hline
\end{tabular}




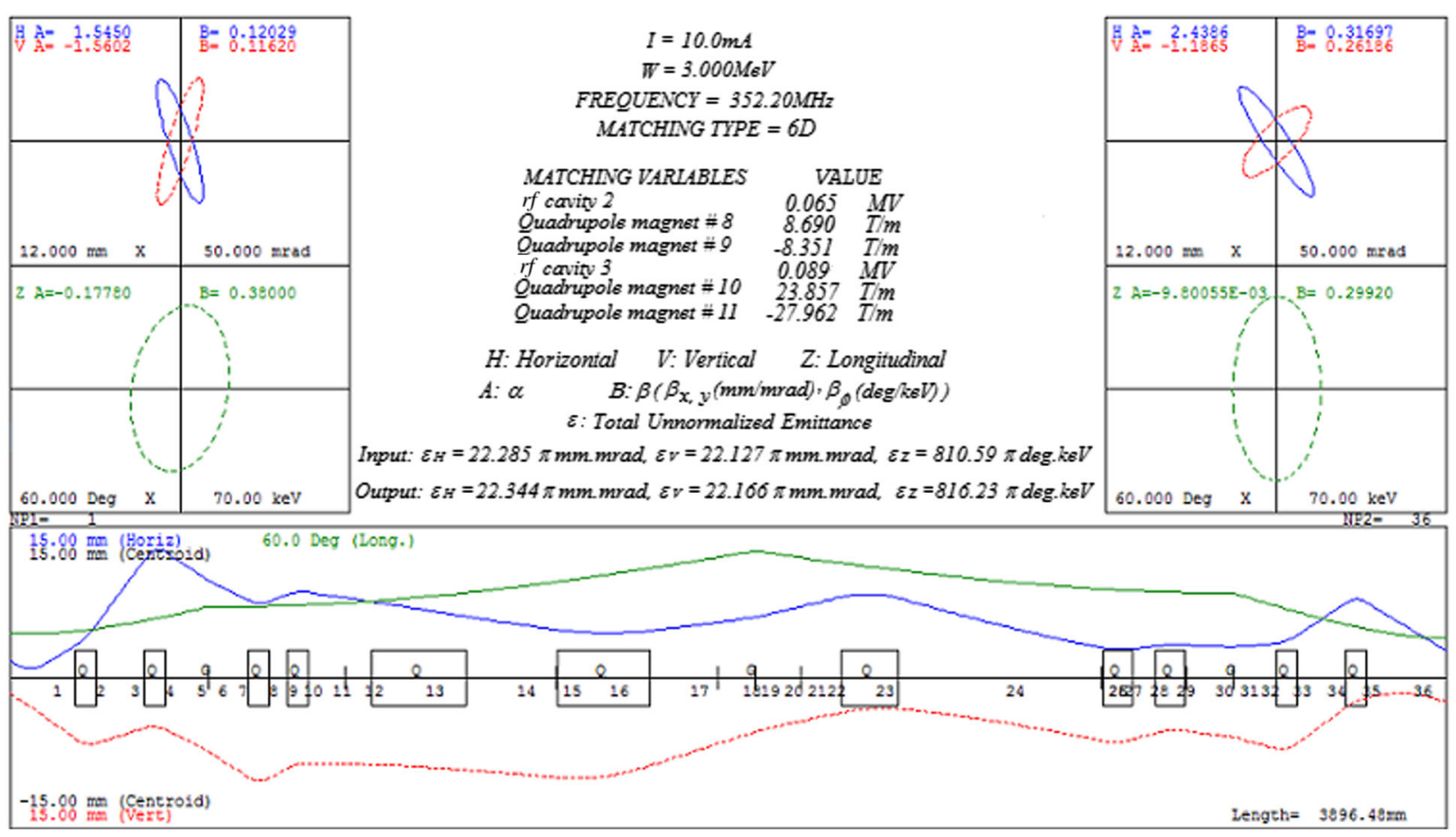

FIG. 11. TRACE 3-D output for matching the $10 \mathrm{~mA}$ RFQ output beam to the DTL by six transverse and longitudinal MEBT knobs. The knob numbers are indicated in Fig. 1.

simulation $94 \%$ of the beam propagates to the end of structure. Therefore, the selected MEBT parameter set was chosen for the beam commissioning of the upstream accelerating structures at a beam current of $10 \mathrm{~mA}$ from the RFQ. The simulation was launched by an empirical beam distribution introduced in Sec. IV and shown in Table III.

Additionally, to verify the performance of the Pareto optimal front and the selected TOPSIS solution from the NSAGII optimization for MEBT, the TRACE 3-D code was applied to solve the same matching problem between the RFQ output beam and the DTL entrance. The result, in Fig. 11 and Table III, shows a good convergence between the solutions found with NSGAII-TOPSIS and TRACE 3-D. The knob values for the Linac4 MEBT matching solution from TRACE 3-D, representing a local optimum, and from the joined NSGAII and TOPSIS method, identifying the global optimum, agree to within less than $10 \%$. The good agreement with TRACE 3-D for this example lends confidence in the use of the NSGAII-TOPSIS as a tool for more complicated matching problems, for correctly balancing multiple design considerations, etc.

The MEBT knobs were set to the NSGAII optimized parameters as the nominal MEBT values for matching the RFQ output beam to DTL. The distribution in the transverse phase spaces was measured at the slit using the slit-and-grid emittance meter located downstream the MEBT output plane and the slit-and-grid emittance measurement has an error of order $\pm 10 \%$ [25,26]. Figure 12 shows the shape of the measured and simulated beam at the slit position and the further details are tabled bellows. It shows a fair agreement between the measured beam distribution and the beam tracked by the code TRAVEL v.04 based on setting of the MEBT component by the TOPSIS and NSGAII optimized parameters. Furthermore in the commissioning of the DTL tanks the beam injected into the DTL has fully transmitted and accelerated [27].

\section{CONCLUSIONS}

The careful matching to each downstream portion of a linear accelerator is important for avoiding envelope mismatch oscillations. We have developed a novel technique, based on the NSGAII genetic algorithm and the TOPSIS selection tool for matching and transporting a beam with consideration of space charge in a linear accelerator.

Specifically, considering the example of the CERN LINAC4, we demonstrated the use and the effectiveness of nondominated sorting genetic algorithm II and technique for order preference by similarity to ideal solution for optimizing the beam matching and transport for a modern hadron linac including the effect of space charge.

Our reported method is easy to implement. We first determine the characteristic beam-envelope matching parameter for each accelerating structure, and then, with the help of the genetic algorithm, minimize the mismatching factors and maximize the beam transmission, while respecting a set of optics constraints. We finally find 


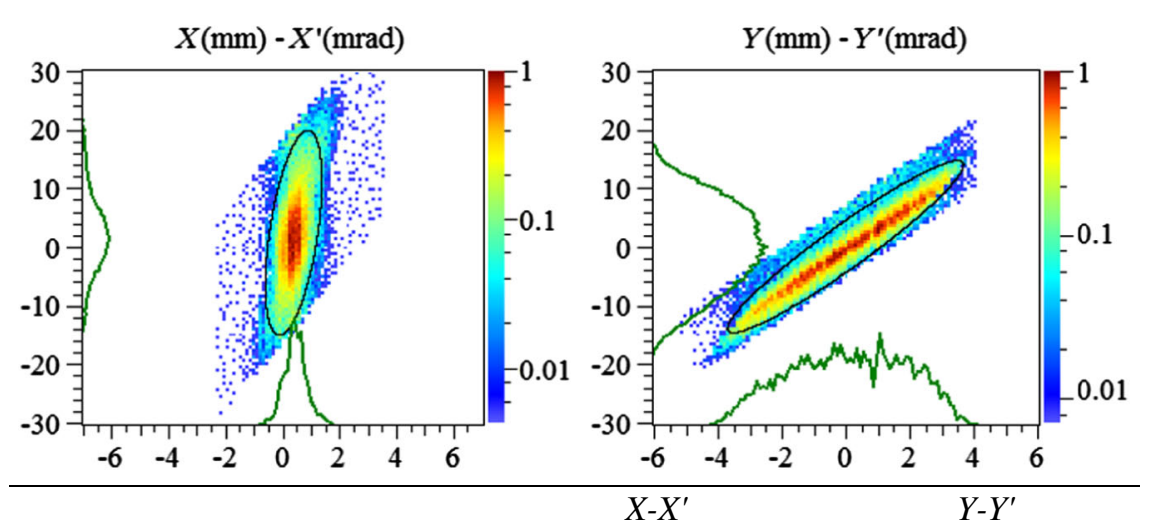

\begin{tabular}{ccc}
\hline Normalized rms Emittance & $0.26 \pi \mathrm{mm} . \mathrm{mrad}$ & $0.31 \pi \mathrm{mm} . \mathrm{mrad}$ \\
\hline$\beta$ & $0.066 \mathrm{~mm} / \pi \mathrm{mrad}$ & $0.83 \mathrm{~mm} / \pi \mathrm{mrad}$ \\
\hline$\alpha$ & -0.59 & -3.13 \\
\hline
\end{tabular}

(a)

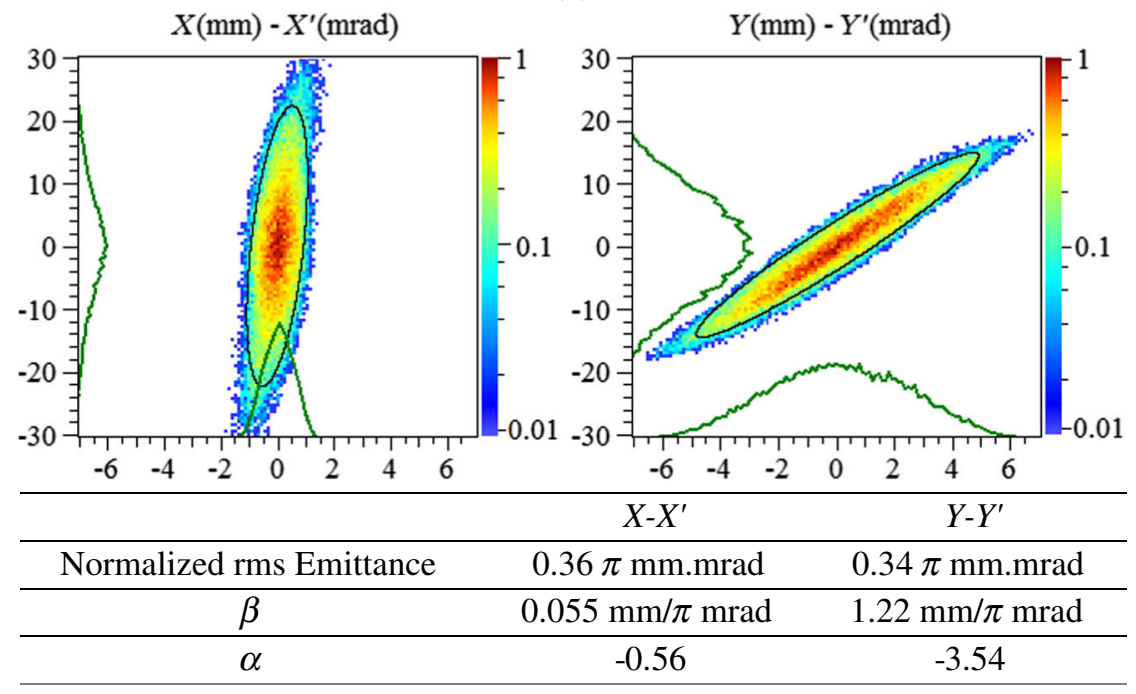

(b)

FIG. 12. (a) Measured beam distribution in the horizontal (left) and vertical (right) phase planes at the slit position on the diagnostic bench [3,25]. (b) Expected horizontal (left) and vertical (right) beam phase space from simulation under the nominal beam matching parameters selected by TOPSIS from NSGAII Pareto optimal front for $10 \mathrm{~mA}$ RFQ output beam current.

our answer at the optimal Pareto front of the solution space by applying TOPSIS.

The selected optimum solution we have found for one part of the LINAC4 machine (MEBT) was independently validated by another space charge code, TRACE 3-D.

We have implemented the solution from the NSGAII and TOPSIS study in the TRAVEL v.04 code. The resulting simulated beam distribution is in good agreement with the transverse phase space distribution measured by a slit-andgrid emittance meter at the input plane of the DTL.

\section{ACKNOWLEDGMENTS}

The authors would like to thank J. B. Lallement, V. Dimov, and C. V. Lizarraga for fruitful discussions. Special thanks go to A. A. Saberi, A. Hosseiny, and
E. Eskandari who have lent their desktop computers for the parallel computational processes. In particular, we warmly thank A. Rouhi for his assistance and support.

[1] F. Gerik, M. Vretenar et al., Linac4 technical design report (CERN Report No. CERN-AB-2006-084 ABP/RF, 2006).

[2] G. Bellodi, A. Dimov, A. Lombardi, F. Zocca, U. Raich, F. Roncarolo, M. Yarmohammadi Satr, and F. Zocca, Longitudinal beam profile measurements in Linac4 commissioning, in Proceedings of 27th International Linear Accelerator Conference, LINAC2014, Geneva, Switzerland, 2014 (JACoW, Geneva, Switzerland, 2014), pp. 108110, http://accelconf.web.cern.ch/AccelConf/LINAC2014/ papers/mopp025.pdf. 
[3] M. Yarmohammadi Satri, G. Bellodi, D. V. J. Lallement, A. Lombardi, F. Roncarolo, and F. Zocca, Transverse beam profile measurement in the Linac4 medium energy beam transport, in Proceedings of 27th International Linear Accelerator Conference, LINAC2014, Geneva, Switzerland, 2014 (JACoW, Geneva, Switzerland, 2014), pp. 516518, http://accelconf.web.cern.ch/AccelConf/LINAC2014/ papers/tupp038.pdfs.

[4] J. B. Lallement, A. Akroh, G. Bellodi, J. F. Comblin, V. A. Dimov, E. Granemann Souza, J. Lettry, A. M. Lombardi, O. Midttun, E. Ovalle, U. Raich, F. Roncarolo, C. Rossi, J. L. Sanchez Alvarez, R. Scrivens, C. A. ValerioLizarraga, M. Vretenar, and M. Yarmohammadi Satri, First commissioning experience with the Linac4 $3 \mathrm{MeV}$ front-end at CERN, in Proceedings of the North American Particle Accelerator Conference, NA-PAC2013, Pasadena, CA, USA, 2013 (JACoW, Geneva, Switzerland, 2013), pp. 52-56, http://accelconf.web.cern.ch/AccelConf/ PAC2013/papers/mozba1.pdf.

[5] M. Vretenar et al., Status and plans for Linac4 instalation and commissioning, in Proceedings of 5th International Particle Accelerator Conference, IPAC2014, Dresden, Germany, 2014 (JACoW, Geneva, Switzerland, 2014), pp. 3332-3334, http://accelconf.web.cern.ch/AccelConf/ IPAC2014/papers/thpme048.pdf.

[6] T. Wangler, RF Linear Acceleration (Wiley, New York, 2008).

[7] A. S. Hofler, Genetic algorithms and their applications in accelerator physics, in Proceedings of the North American Particle Accelerator Conference, NA-PAC2013, Pasadena, CA, USA, 2013 (JACoW, Geneva, Switzerland, 2013), pp. 1111-1115, http://accelconf.web.cern.ch/ AccelConf/PAC2013/papers/thtb1.pdf.

[8] A. Hofler, B. Terzic, M. Kramer, A. Zvezdin, V. Morozov, Y. Roblin, F. Lin, and C. Jarvis, Innovative applications of genetic algorithms to problems in accelerator physics, Phys. Rev. ST Accel. Beams 16, 010101 (2013).

[9] W. Gao, L. Wang, and W. Li, Simultaneous optimization of beam emittance and dynamic aperture for electron storage ring using genetic algorithm, Phys. Rev. ST Accel. Beams, 14, 094001 (2011).

[10] I. V. Bazarov and C. K. Sinclair, Multivariant optimization of a high brightness dc gun photoinjector, Phys. Rev. ST Accel. Beams 8, 034202 (2005).

[11] C. Sun, D. S. Robin, H. Nishimura, C. Steier, and W. Wan, Small-emittance and low-beta lattice design and optimizations, Phys. Rev. ST Accel. Beams 15, 054001 (2012).

[12] L. Bonacina, J. Extermann, A. Rondi, V. Boutou, and J.-P. Wolf, Multiobjective genetic approach for optimal control of photoinduced processes, Phys. Rev. A 76, 023408 (2007).

[13] S. Yarmohammadisatri, M. H. Shojaeefard, and A. Khalkhali, A family base optimization of a developed nonlinear vehicle suspension model using gray family design algorithm, Nonlinear Dyn. 90, 649 (2017).

[14] M. H. Shojaeefard, K. Abolfazl, and S. Yarmohammadisatri, An efficient sensitivity analysis method for modified geometry of Macpherson suspension based on Pearson correlation coefficient, Vehicle System Dynamics 55, 827 (2017).

[15] R. Talebitooti, M. Shojaeefard, and S. Yarmohammadisatri, Shape design optimization of cylindrical tank using b-spline curves, Computers Fluids 109, 100 (2015).

[16] K. R. Crandall and D. P. Rusthoi, TRACE 3-D Documentation, 3rd ed. (Los Alamos National Laboratory, Los Alamos, New Mexico, 1997).

[17] M. G. Minty and F. Zimmermann, Measurement and Control of Charged Particle Beams (Springer-Verlag, Berlin, 2003).

[18] K. Deb, A. Pratap, S. Agarwal, and T. Meyarivan, A fast and elitist multiobjective genetic algorithm: NSGA-II, IEEE Trans. Evol. Comput. 6, 182 (2002).

[19] A. Perrin, J. F. Amand, T. Mutze, J. B. Lallement, and S. Lanzone, TRAVEL v4.06: User Manual, CERN Internal Note, 2007.

[20] J. B. Lallement, G. Bellodi, V. A. Dimov, A. Lombardi, and M. Yarmohammadi Satri, Linac4 transverse and longitudinal emittance reconstruction in the presence of space charge in Proceedings of 27th International Linear Accelerator Conference, LINAC2014, Geneva, Switzerland, 2014 (JACoW, Geneva, Switzerland, 2014), pp. 913915, http://accelconf.web.cern.ch/AccelConf/LINAC2014/ papers/thpp033.pdf.

[21] C. L. Hwang and K. P. Yoon, Multiple attribute decision making: Method and applications (Springer-Verlag Press, Heidelberg, 1981).

[22] K. Paul Yoon and W. K. Kim, The behavioral TOPSIS, Expert Systems with Applications 89, 266 (2017).

[23] R. Scrivens et al., Linac4 low energy beam measurements with negative hydrogen ions, Rev. Sci. Instrum. 85, 02A729 (2014).

[24] T. Mütze and S. Lanzone, Delta User Manual, CERN Internal Note, 2007.

[25] A. M. Lombardi, Commissioning of the low-energy part of Linac4, in Proceedings of 27th International Linear Accelerator Conference, LINAC2014, Geneva, Switzerland, 2014 (JACoW, Geneva, Switzerland, 2014), pp. 610, http://accelconf.web.cern.ch/AccelConf/LINAC2014/ papers/moioa02.pdf.

[26] F. Roncarolo et al., Transverse profile and emittance measurements with a laser stripping system during the cern Linac4 commissioning at 3 and $12 \mathrm{MeV}$, in LINAC14: Proceedings of the 27th International Linear Accelerator Conference, Geneva, Switzerland, 2014 (JACoW, Geneva, Switzerland, 2014), pp. 506-509, http://accelconf.web .cern.ch/AccelConf/LINAC2014/papers/tupp035.pdf.

[27] V. A. Dimov, E. Belli, G. Bellodi, J. B. Lallement, A. M. Lombardi, and M. Yarmohammadi Satri, Beam commissioning of Linac4 up to $12 \mathrm{MeV}$ in Proceedings of 6th International Particle Accelerator Conference, IPAC2015, Newport News, VA, USA, 2015 (JACoW, Geneva, Switzerland, 2015), pp. 2408-2412, http://accelconf.web.cern.ch/ AccelConf/IPAC2015/papers/weyb2.pdf. 